\title{
Tsunami generation by ocean floor rupture front propagation: Hamiltonian description
}

\author{
V. I. Pavlov ${ }^{1,2}$, J. Tromp ${ }^{1}$, and E. P. Tito \\ ${ }^{1}$ California Institute of Technology, Mail Code 252-21, Pasadena, California 91125, USA \\ ${ }^{2}$ UFR de Mathématiques Pures et Appliquées - LML UMR 8107, Université de Lille 1, 59655 Villeneuve d'Ascq, France
}

Received: 18 July 2008 - Revised: 16 January 2009 - Accepted: 16 January 2009 - Published: 23 February 2009

\begin{abstract}
The Hamiltonian method is applied to the problem of tsunami generation caused by a propagating rupture front and deformation of the ocean floor. The method establishes an alternative framework for analyzing the tsunami generation process and produces analytical expressions for the power and directivity of tsunami radiation (in the farfield) for two illustrative cases, with constant and gradually varying speeds of rupture front propagation.
\end{abstract}

\section{Introduction}

A tsunami is generated when the ocean floor abruptly deforms and vertically displaces overlying water. Generally speaking, any strong disturbance of the ocean resulting from seismic motions, asteroid impacts, volcanic eruptions, gas releases, submarine landslides, etc. that displaces a large water mass from its equilibrium position can be the source of a tsunami. A number of publications review this topic extensively (see, for example, Keating et al., 2000; Bryant, 2001; Ward et al., 2003; Kanamory and Brodsky, 2004; Titov et al., 2005; Harbitz et al., 2006; Wiegel, 2006; Gisler, 2008). The physics of the process of wave generation by a propagating deformation of the sea floor is rather transparent and can be studied in linear approximation with a number of methods (see, for example, Novikova and Ostrovsky (1979); Nosov (1998) and the references therein). Often tsunami generation is described by a piston mechanism of ocean floor motion. Such a model assumes that a tsunami is generated as a result

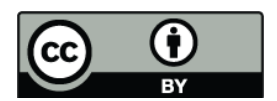

Correspondence to: V. I. Pavlov (vpavlov@caltech.edu) of a quick rise of the sea bottom occurring uniformly or nonuniformly (Hatori, 1970; Murty, 1977; Tanioka and Satake, 1996) in the focal area of the earthquake. The water, pushed upward from its equilibrium position, attempts to regain its equilibrium under the influence of gravity and causes surface wave propagation. While simple and convenient, the piston mechanism has its limitations because it does not fully capture the entire set of complex tsunami characteristics (see, for example, Tinti and Bortolucci, 2000; Tinti et al., 2001).

In this paper we apply the Hamiltonian method to the tsunami generation problem, which is an alternative to the traditional approach. Specifically, we analyze the radiation effect caused by a long-duration ocean floor rupture front propagation, in the far-field, for both constant and varying rupture speeds.

We want to emphasize that typically it is the phenomenon that is the focal point of research. Thus, most studies describe and analyze specific events, effects, or models. In this paper, while considering a specific phenomenon, we focus on dealing with the method rather than the phenomenon. We aim to develop a methodology that is applicable to a broad range of cases in a general way. To do so, we naturally start with simple and somewhat idealized cases. However, once the method is established, its ability to consider a variety of circumstances should serve as a valuable tool for seismological research.

The Hamiltonian method is one of the most advanced and powerful analytical methods for working with problems involving wave radiation and interactions. It became popular after the pioneering papers by Zakharov and Filonenko (1966) for surface waves applications, and now is frequently used as an operational tool (see, for example, Lavrenov, 2003; Jansen, 2004). The description of versions of the

Published by Copernicus Publications on behalf of the European Geosciences Union. 
approach in the general physical context of wave and vortex motions can be found, for example, in books and reviews by Goncharov and Pavlov (1993, 1997); Zakharov and Kuznetsov (1997); Goncharov and Pavlov (1998, 2000, 2001, 2008) and the extensive bibliographies therein.

Our work was in part motivated by experimental data showing that the rupture fronts of earthquakes (which when occurring underneath the ocean generate a tsunami by deforming the ocean floor) propagate with varying speeds, and with patterns not consistent with the piston model. Seismic radiation data collected after large earthquakes have provided vast material for mapping and numerically simulating earthquake rupture processes (Dahlen and Tromp, 1998). Data from the 2004 Sumatra-Andaman earthquake (Ammon et al., 2005) showed that the deformation of the floor surface propagated with a speed which varied in magnitude and direction over an extended period of time (more than $10 \mathrm{~min}$ ) and great distance $(\sim 1500 \mathrm{~km})^{1}$. Data from the 2001 Kunlun, China, earthquake showed rupture velocities exceeding the shear wave speed, while the 1994 Bolivian earthquake's rupture speeds were only about one fifth of the shear wave speed (Kanamory and Brodsky, 2004). Arial photography after the Alaskan 1964 earthquake showed the most dramatic damage on the ground aggregated along zigzaging trajectories (Ivanov, 1991) as if the source of the earthquake (rupture front) traveled underneath the surface with fast and directionally varying velocity, resulting in a tangled destruction pattern on the surface. The fact that rupture velocities range so broadly means that the general theory describing the process must be comprehensive enough to cover the entire spectrum. The method explained in this paper possesses such capabilities.

In order to capture the first-order attributes of the process of tsunami generation and to illustrate the features of the method, we consider two simple models where the deformation of the ocean floor travels for sufficiently long time with (a) constant and (b) varying speed. The rupture front can be viewed as a "moving source" generating waves. By making this analogy, we place the problem of tsunami generation into the framework developed for general physical problems of wave generation by moving sources (Pavlov and Sukhorukov, 1985, 1987; Pavlov and Slabeycius, 1985; Ginzburg, 1996). We use the simplest approximation which is similar to the "slender body" model (which in hydrodynamic equations approximates the real submerged body as a source with fixed characteristics). Indeed, the general physical picture of the radiated wave field generated by a moving ship or by a moving (at long distances) ocean floor fault is qualitatively the same (Sretensky, 1977). This is not surprising because the problem involves the general physical phenomenon of superposition and phasing of emitted waves. The difference

\footnotetext{
${ }^{1}$ See http://mr.caltech.edu/media/Press_Releases/PR12698.html for an animation of the sea floor displacement and its evolution in time and space, constructed based on the recorded seismic data.
}

between the ship and the fault is determined by the formfactor structure of the boundary surface perturbation which gives information about the stress. Here, we would like to emphasize the fact that the radiated field forms not in the point where the source is located, but is "assembled" from the fields generated by all points along the entire trajectory (Pavlov and Sukhorukov, 1985). Therefore, phase effects for the waves superposing at the point of observation play the most critical role.

The remainder of this short paper is organized as follows. In Sect. 2 we define our model and method (the Hamiltonian approach). In Sect. 3, we apply the Green function method with functional derivatives which allows us to analyze wave propagation, calculate wave characteristics and derive the angular-spectral power of the radiation for a moving source. In Sect. 4, we consider a model with a narrow floor rupture moving rapidly with constant velocity. In Sect. 5, we consider radiation from a localized rupture front propagating with smoothly varying velocity. Section 6 summarizes the results. Appendices A and B discuss made approximations in more detail.

\section{The Hamiltonian approach for surface waves}

It is known (Zakharov and Filonenko, 1966; Goncharov and Pavlov, 1993, 1998; Zakharov, 1999) that the potential motion of a fluid with free boundary is completely described by the surface elevation $\eta(\boldsymbol{x}, t)$ and a velocity potential on the surface $\psi(\boldsymbol{x}, t)=\phi(\boldsymbol{x}, z=\eta, t)$, which are also canonical variables. These quantities satisfy the equations

$\partial_{t} \eta=\frac{\delta H}{\delta \psi}, \quad \partial_{t} \psi=-\frac{\delta H}{\delta \eta}$.

We assume the standard geometry of the model: the unperturbed fluid surface coincides with the $x-y$-plane, such that the surface of the ocean is at $z=0$ and the non-perturbed bottom is at $z=D$, the $z$-axis points vertically up and the gravity acceleration $\boldsymbol{g}$ is directed vertically down. Symbol $\delta F[u] / \delta u(\boldsymbol{x}, t)$ defines the variational derivative of a functional with respect to field $u(\boldsymbol{x}, t)$. Here, we introduce the Cartesian coordinate system, $\mathbf{r}=(\boldsymbol{x}, z)$. The Hamiltonian $H$ is the full energy of the fluid

$H=\int d \boldsymbol{r}\left[\Pi(\rho)+\rho g z+\rho \frac{\boldsymbol{v}^{2}}{2}\right]+H_{\Sigma} \equiv H[\rho, \nabla \phi]$.

Here, $\Pi(\rho)$ is the internal energy of the fluid with density $\rho, g$ is gravity acceleration, $v$ is velocity of the fluid particles. The term $H_{\Sigma}=\rho \sigma \int d \boldsymbol{x}\left[\sqrt{1+(\nabla \eta)^{2}}-1\right]$ describes surface energy when the surface tension $\sigma$ and the curvature of surface are not neglected. The potential flow is $\boldsymbol{v}=\nabla \phi$. The generalized density is defined by the expression $\rho[\eta]=\rho_{0} \theta(\eta-z) \theta(z+d)$, were $\theta$ is the Heaviside function: $\theta(s)=1$ if $s \geq 0$ and $\theta(s)=0$ if $s<0$. The function $d=d(\boldsymbol{x}, t)$ describes the bottom perturbations, $\eta=\eta(\boldsymbol{x}, t)$ characterizes 
the perturbed ocean surface. The Hamiltonian given by Eq. (2) can be decomposed into the sum $H=H_{f}+H_{\text {int }}$, where the first term is the field part of the Hamiltonian, and the second describes the source (i.e. the interaction of the fluid with the mobile part of the bottom). The field Hamiltonian $H_{f}$ can be presented by a power series expansion in field magnitudes: $H_{f}=H_{2}+H_{3}+H_{4}+\ldots$ where $H_{n} \sim 0\left(\eta^{n}\right)$. After transitioning to surface variables, the Hamiltonian $\mathrm{H}_{2}$ (describing non-interacting surface waves) is expressed as

$H_{2}=\frac{1}{2} \int d x\left[\eta\left(g+\sigma \widehat{\Gamma}^{2}\right) \eta+\psi \widehat{\Gamma} \tanh D \widehat{\Gamma} \psi\right]$.

In the linear approximation, the equations are

$$
\begin{array}{r}
\partial_{t} \eta=\frac{\delta H_{2}}{\delta \psi}=\hat{\Gamma} \tanh D \hat{\Gamma} \psi, \\
\partial_{t} \psi=-\frac{\delta H_{2}}{\delta \eta}=-g \eta-\sigma \hat{\Gamma}^{2} \eta .
\end{array}
$$

The term with the coefficient of surface tension $\sigma$ appears when we transition to surface variables and take into account the dynamical conditions at the free surface. Henceforth we assume $\rho=1$. This decision implies that the dimension of the Hamiltonian changes from $M L^{2} T^{2}$ to $L^{5} T^{2}$. The operator $\widehat{\Gamma}=\sqrt{-\Delta}$ is a linear integral (pseudo-differential) operator, defined in Fourier space as

$\widehat{\Gamma} \eta=\int \frac{d \boldsymbol{k}}{2 \pi}|\boldsymbol{k}| \eta_{\boldsymbol{k}} e^{i \boldsymbol{k} \cdot \boldsymbol{x}}$,

i.e., $\hat{\Gamma}$ acts on the exponent as $\hat{\Gamma} e^{i \boldsymbol{k} \cdot \boldsymbol{x}}=|\boldsymbol{k}| e^{i \boldsymbol{k} \cdot \boldsymbol{x}}$.

In terms of normal variables (Zakharov, 1999), the Hamiltonian $\mathrm{H}_{2}$ takes the form

$H_{2}=\int d \boldsymbol{k} \omega_{k} a_{k} a_{k}^{*}$,

The normal variables express the surface $\eta(\boldsymbol{x}, t)$ and the velocity potential at the surface $\psi(\boldsymbol{x}, t)=\phi(\boldsymbol{x}, z=\eta(\boldsymbol{x}, t), t)$ as

$$
\begin{array}{r}
\eta(\boldsymbol{x}, t)=\int \frac{d \boldsymbol{k}}{2 \pi} \sqrt{\frac{\omega_{\boldsymbol{k}}}{2 g_{k}}}\left(a_{\boldsymbol{k}}+a_{-\mathbf{k}}^{*}\right) e^{i \boldsymbol{k} \cdot \boldsymbol{x}}, \\
\psi(\boldsymbol{x}, t)=\int \frac{d \boldsymbol{k}}{2 \pi}(-i) \sqrt{\frac{g_{k}}{2 \omega_{\boldsymbol{k}}}}\left(a_{\boldsymbol{k}}-a_{-\boldsymbol{k}}^{*}\right) e^{i \boldsymbol{k} \cdot \boldsymbol{x}} .
\end{array}
$$

Here, $\omega_{\boldsymbol{k}}=\sqrt{k g_{k} \tanh k D}$ is the dispersion law for free waves, $\boldsymbol{k}$ is the wave vector, $k=|\boldsymbol{k}|$ is the magnitude of $\boldsymbol{k}$, $D$ is the thickness of the water layer, $g_{k}=g+\sigma k^{2}, g$ is the gravitational acceleration, $\sigma$ is the surface tension coefficient. Capillary effects can be neglected when energy emission is concerned, since the energy-carrying component of the surface wave is localized in the large-scale region of the spectrum, i.e. when wave-numbers of the components are small, $k \ll \sqrt{g / \sigma}$. However, capillary waves can play an important role when the "shock" surface wave forms. Also, the presence of the capillary term allows for the mathematical convergence of certain integrals in the derivations we are pursuing. Therefore, we will not omit this term right away in our calculations ${ }^{2}$.

We can show ${ }^{3}$ that the Hamiltonian of interaction is

$H_{\text {int }}=-\frac{1}{2} \int d \boldsymbol{x} \phi(\boldsymbol{x},-D, t) \partial_{t} d\left(\boldsymbol{x}-\boldsymbol{x}_{0}(t)\right)$.

Here, $d$ describes the ocean floor deformation traveling with speed $\dot{\boldsymbol{x}}_{0}(t)$. The velocity potential is calculated at the nonperturbed bottom because in the Hamiltonian we take into account only the leading terms.

The structure of the Hamiltonian of interaction may be established from simple qualitative reasoning even without detailed calculations. The Hamiltonian has dimension $L^{5} T^{-2}$. The dimension of the integration is $\left[\int d \boldsymbol{x} \ldots\right]=L^{2}$. The dimensions of the displacement of the ocean bottom $d$ and of the field variable $\phi$ are $[d]=L$ and $[\phi]=L^{2} T^{-1}$, respectively. The first term in the power series expansion of the Hamiltonian functional has to contain these quantities to assure the linearity of the problem. Thus, we may propose the following structure

$$
\left.H_{\mathrm{int}} \sim \int d \boldsymbol{x} \phi(\boldsymbol{x}, z, t)\right|_{z=-D} \widehat{L} d(\boldsymbol{x}, t),
$$

where $\widehat{L}$ is some linear differential operator acting on one of the field variables and assuring the locality of the interaction. Comparing the dimensions of the right and left parts, we find that $\widehat{L}$ is proportional to the partial derivative with respect to time, $\partial_{t}$, and thus derive Eq. (8). A detailed analysis gives the same expression to within a numerical coefficient of order of one.

\section{Power of radiation}

The radiation intensity of waves (generated by a moving source) on the surface of a perfect fluid can by calculated with comparative ease if the equations of motion for the fluid are written in the form

$$
\begin{gathered}
\partial_{t} a_{\boldsymbol{k}}=-i \frac{\delta H}{\delta a_{\boldsymbol{k}}^{*}}=-i \omega_{\boldsymbol{k}} a_{\boldsymbol{k}}-i \frac{\delta H_{\mathrm{int}}}{\delta a_{\boldsymbol{k}}^{*}}, \\
\partial_{t} a_{\boldsymbol{k}}^{*}=+i \frac{\delta H}{\delta a_{\boldsymbol{k}}}=+i \omega_{\boldsymbol{k}} a_{\boldsymbol{k}}^{*}+i \frac{\delta H_{\mathrm{int}}}{\delta a_{\boldsymbol{k}}} .
\end{gathered}
$$

To describe the bottom-fluid interaction, we introduce the Green function $G\left(t, t^{\prime}\right)=\theta\left(t-t^{\prime}\right) e^{-i \omega_{k}\left(t-t^{\prime}\right)}$, which satisfies the equation

$$
\partial_{t} G+i \omega_{k} G=\delta\left(t-t^{\prime}\right) .
$$

\footnotetext{
${ }^{2}$ The wavenumber domain of capillary waves is important for observations from space using inverse scattering of centimeter radiowaves from the excited ocean surface.

${ }^{3}$ The volume integral describing kinetic energy can be transformed into a surface integral at the free surface and the perturbed floor. The Hamiltonian of interaction, with the boundary conditions taken into consideration, represents specifically this part.
} 
We can the rewrite Eq. (10) in integral form

$a_{k}(t)=e^{-i \omega_{k} t} \int_{-\infty}^{+\infty} d t^{\prime} \theta\left(t-t^{\prime}\right)\left[-i \frac{\delta H_{\mathrm{int}}}{\delta a_{\boldsymbol{k}}^{*}(t)}\right] e^{+i \omega_{\boldsymbol{k}} t^{\prime}}$.

Using Eq. (12), we find the spectral density of radiated energy of surface waves:

$$
\begin{array}{r}
\left.\left.E_{\boldsymbol{k}}\right|_{t \rightarrow \infty} \equiv \omega_{\mathbf{k}} a_{\boldsymbol{k}} a_{\boldsymbol{k}}^{*}\right|_{t \rightarrow \infty} \rightarrow \\
\rightarrow \omega_{\boldsymbol{k}}\left|\int_{-\infty}^{+\infty} d t^{\prime} e^{-i \omega_{\boldsymbol{k}} t^{\prime}} \frac{\delta H_{\mathrm{int}}}{\delta a_{\boldsymbol{k}}}\right|^{2} .
\end{array}
$$

All information describing the radiation intensity is contained in the Hamiltonian of interactions $H_{\text {int }}$.

The velocity potential is the solution of the problem $\Delta \phi=0$ with boundary conditions $\left.\phi\right|_{z=0}=\psi$ and $\left.\partial_{z} \phi\right|_{z=-D}=0$. It is satisfied by the expression

$\phi(\boldsymbol{x}, z, t)=\int \frac{d \boldsymbol{k}}{2 \pi} \psi(\boldsymbol{k}) \frac{\cosh k(z+D)}{\cosh k D} e^{i \boldsymbol{k} \cdot \boldsymbol{x}}$.

Now we calculate the derivatives

$$
\begin{array}{r}
\left.\frac{\delta \phi}{\delta a_{\boldsymbol{k}}}\right|_{z=-D}=\frac{\delta}{\delta a_{\boldsymbol{k}}} \int \frac{d \boldsymbol{q}}{(2 \pi)}(-i) \frac{1}{\cosh q D} \sqrt{\frac{g_{q}}{2 \omega_{\boldsymbol{q}}}} \\
\times\left(a_{\boldsymbol{q}}-a_{-\boldsymbol{q}}^{*}\right) e^{i \boldsymbol{q} \cdot \boldsymbol{x}}= \\
=\frac{1}{i 2 \pi} \frac{1}{\cosh k D} \sqrt{\frac{g_{k}}{2 \omega_{\boldsymbol{k}}}} e^{i \boldsymbol{k} \cdot \boldsymbol{x}} .
\end{array}
$$

From here, the expression for the derivative corresponding to a source traveling with uniform speed $\boldsymbol{V}$ follows as

$$
\begin{array}{r}
\frac{\delta H_{\mathrm{int}}}{\delta a_{\boldsymbol{k}}}=-\left.\int d \boldsymbol{x} \partial_{t} d(\boldsymbol{x}-\boldsymbol{V} t) \frac{\delta}{\delta a_{\boldsymbol{k}}} \phi(\boldsymbol{x}, z, t)\right|_{z=-D}= \\
=\frac{i}{2 \pi} \frac{1}{\cosh k D} f_{\boldsymbol{k}}^{*} \sqrt{\frac{g_{k}}{2 \omega_{\boldsymbol{k}}}} e^{+i \boldsymbol{k} \cdot \boldsymbol{V} t} .
\end{array}
$$

The parameter $f_{k}$ is the form-factor of the bottom perturbation:

$f_{\boldsymbol{k}}^{*}=\int d \boldsymbol{x}^{\prime} \partial_{t} d\left(\mathbf{x}^{\prime}\right) e^{+i \boldsymbol{k} \cdot \boldsymbol{x}^{\prime}}$.

In this case, the spectral energy density of surface wave radiation is

$$
=\frac{g_{k}}{2(2 \pi)^{2} \cosh ^{2} k D}\left|\int_{-\infty}^{+\infty} d t^{\prime} f_{\boldsymbol{k}} e^{-i\left(\omega_{\boldsymbol{k}}-\boldsymbol{k} \cdot \boldsymbol{V}\right) t^{\prime}}\right|^{2} .
$$

\section{Model with a narrow ocean floor rupture}

Now let us consider a specific expression for the deformation of the ocean floor. To obtain simple but transparent analytical estimates, we consider the simplest model: a narrow bottom deformation, $l_{\perp}$ wide and $\epsilon D$ deep, traveling with uniform rupture speed $V$ along the $x$-axis. Thus we assume the shape of the vertical displacement of the ocean floor to be

$d(\boldsymbol{x}, t)=D\left[1+\epsilon \delta^{(1)}\left(\frac{y}{l_{\perp}}\right) \theta^{(1)}\left(\frac{V t-x}{l_{\|}}\right)\right]$.

Here, $l_{\perp}$ is the characteristic transversal width of the rupture, $l_{\|}$is its characteristic longitudinal scale indicating the slope of the rupture depth (from 0 to $-\epsilon D), \delta^{(1)}(\xi)$ is the Dirac function indicating that floor deformation is narrow, $\theta^{(1)}(\xi)$ is the Heaviside function, i.e. $\partial_{\xi} \theta^{(1)}(\xi)=\delta^{(1)}(\xi)$. The use of the Dirac function in the estimates is admissible when the spectral maximum of emitted surface waves is in the lowfrequency range. This assumption will be supported by the calculations that follow. The parameter $\epsilon$ is the dimensionless (normalized with respect to $D$ ) magnitude of the floor perturbation. It can be a function of time in some cases when it is necessary to take into account the finite duration of the faulting process. For example, $\epsilon$ can be replaced by $\epsilon \rightarrow \epsilon \exp \left(-t^{2} / \tau^{2}\right)$ or by $\epsilon \theta(t) \exp (-t / \tau)$, where $\tau$ is a characteristic temporal scale of the process duration.

If $\epsilon$ is constant, from Eq. (19) we find

$$
\begin{aligned}
\partial_{t} d= & \epsilon D \frac{V}{l_{\|}} \delta^{(1)}\left(\frac{y}{l_{\perp}}\right) \delta^{(1)}\left(\frac{x-V t}{l_{\|}}\right)= \\
& =\epsilon D V l_{\perp} \delta^{(1)}(y) \delta^{(1)}(x-V t) .
\end{aligned}
$$

Having

$\partial_{t} d\left(\boldsymbol{x}^{\prime}\right)=\epsilon D V l_{\perp} \delta^{(2)}\left(\boldsymbol{x}^{\prime}\right)$,

we find from Eq. (17) that

$$
f_{\boldsymbol{k}}^{*}=\epsilon D V l_{\perp} \quad \rightarrow \quad\left|f_{\boldsymbol{k}}\right|^{2}=(\epsilon D)^{2} V^{2} l_{\perp}^{2} .
$$

Here, $f_{\boldsymbol{k}}^{*}$ is real, however, in general the form-factor is defined to within a phase factor. Collecting all the formulae, we find the expression for the spectral energy density of surface wave radiation:

$$
\begin{array}{r}
\left.\left.E_{\boldsymbol{k}}\right|_{t \rightarrow \infty} \equiv \omega_{\mathbf{k}} a_{\boldsymbol{k}} a_{\boldsymbol{k}}^{*}\right|_{t \rightarrow \infty} \rightarrow \\
\rightarrow \omega_{\boldsymbol{k}}\left|\int_{-\infty}^{+\infty} d t^{\prime} e^{-i \omega_{\boldsymbol{k}} t^{\prime}} \frac{\delta H_{\text {int }}}{\delta a_{\boldsymbol{k}}}\right|^{2}= \\
=\frac{\omega_{\boldsymbol{k}}}{(2 \pi)^{2}} \frac{g_{k}}{2 \omega_{\boldsymbol{k}}} \frac{\left|f_{\boldsymbol{k}}\right|^{2}}{\cosh ^{2} k D}\left|\int_{-\infty}^{+\infty} d t^{\prime} e^{-i\left(\omega_{\boldsymbol{k}}-\boldsymbol{k} \cdot \boldsymbol{V}\right) t^{\prime}}\right|^{2} .
\end{array}
$$

By computing the square of the integral modules in Eq. (23), we obtain an expression equal to $(2 \pi)^{2} \delta^{2}(a)$. Here, $\delta^{2}(a)$ is the square of delta function with argument $a \equiv \omega_{\boldsymbol{k}}-\boldsymbol{k} \cdot \boldsymbol{V}$. Following Landau (1965), we can rewrite the square of delta function as $\delta^{2}(a)=\delta(a) \times(2 \pi)^{-1} \lim _{T \rightarrow \infty} \int_{-T / 2}^{+T / 2} d t e^{i a t}$ by decomposing one of the delta functions into the Fourier integral. Because of the delta function presence, the argument in the exponential can be written as zero, i.e. the exponential gets replaced by 1 . Thus, $\delta^{2}(a)=\delta(a) \lim _{T \rightarrow \infty} \frac{T}{2 \pi}$, i.e. 
$E \sim \delta\left(\omega_{\boldsymbol{k}}-\boldsymbol{k} \cdot \boldsymbol{V}\right) \lim _{T \rightarrow \infty} T$, but the infinite time $T$ vanishes when we consider the energy radiated per unit of time, $\dot{E}$. This result has a simple physical meaning: if a body travels infinitely long, it radiates an infinite amount of energy, but the energy radiated per unit of time (power) is obviously finite and physically meaningful. The spectral power of radiation then becomes

$\left.\dot{E}_{\boldsymbol{k}}\right|_{t \rightarrow \infty}=\frac{g_{k}}{4 \pi} \frac{\left|f_{\boldsymbol{k}}\right|^{2}}{\cosh ^{2} k D} \delta^{(1)}\left(\omega_{\boldsymbol{k}}-\boldsymbol{k} \cdot \boldsymbol{V}\right)$.

The process of radiation is therefore similar to the Cherenkov effect: Radiation takes place when the argument of the Dirac function is zero. In acoustics, this phenomenon has the following interpretation: When physical objects without eigenfrequencies move uniformly through homogeneous elastic media, the only possible form of acoustic radiation is the emission of Mach waves which appear at ultrasonic source velocities. A similar effects is observed in hydrodynamics (wave generation by ships, Sretensky, 1977).

We substitute Eq. (22) into the obtained expression and find that

$$
\begin{aligned}
\left.\dot{E}_{\boldsymbol{k}}\right|_{t \rightarrow \infty} & =\frac{1}{4 \pi}(\epsilon D)^{2} l_{\perp}^{2} V^{2} \frac{g_{k}}{\cosh ^{2} k D} \times \\
& \times \delta^{(1)}\left(\sqrt{k g_{k} \tanh k D}-k V \cos \theta\right) .
\end{aligned}
$$

The $\cosh k D$ term favors long waves that contribute the most to the tsunami. Integrating with respect to $k$ (since $d \boldsymbol{k}=d \theta d k k$ ) from 0 to $\infty$, we obtain the angular radiation power

$$
\begin{aligned}
P(\theta)=\frac{1}{4 \pi} & (\epsilon D)^{2} l_{\perp}^{2} V^{2} \int_{0}^{+\infty} d k k \frac{g_{k}}{\cosh ^{2} k D} \times \\
& \times \delta^{(1)}\left(\sqrt{k g_{k} \tanh k D}-k V \cos \theta\right) .
\end{aligned}
$$

Here, $\theta$ is the angle between the velocity vector of rupture propagation and the direction towards the point of observation. The expression is not zero if the argument of the Dirac's function is zero,

$\sqrt{k g_{k} \tanh k D}-k V \cos \theta=0$.

Let $k^{(m)}(\theta)$ be solutions of Eq. (27). Finding the roots of the equation can be visualized using a graph of function $y_{1}(x)$ when Eq. (27) is rewritten in the equivalent form

$y_{1}(x) \equiv\left(1+\frac{x^{2}}{\mu^{2}}\right) \frac{\tanh x}{x}=M^{2} \cos ^{2} \theta \equiv y_{2}$.

Here $\quad x=k D, \mu=k_{m} D, k_{m}=\sqrt{\sigma / g}, \quad M=V / c, c=\sqrt{g D}$. Near the origin function $y_{1}(x)$ starts with $y_{1}(0)=1$. It reaches its minimum value $y_{1}\left(x_{\min }\right)=y_{1}$, min at $x_{\min }$ because $\mu \gg 1$, and then increases tending asymptotically to $y_{1}(x)=x / \mu^{2}$. Value $x_{\min }$ is of order of $\mu$, i.e. $k_{\min } \sim k_{m}$. (For pure water, it corresponds to the phase speed of capillarygravity waves of order $c_{m} \sim 23 \mathrm{~cm} / \mathrm{s}$.) Thus, values $x>x_{\min }$ correspond to capillary waves, while $x<x_{\min }$ to gravity

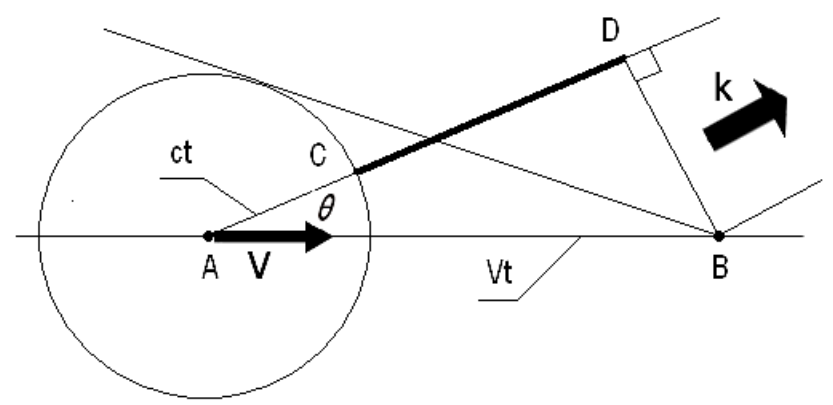

Fig. 1. Phasing condition for Cherenkov radiation.

waves. The intersection of function $y_{2}$ (which is not $x$ dependent and is shown by the horizontal line on the graph) with $y_{1}(x)$ determines the roots of Eq. (27). It is obvious that if $y_{2} \equiv M^{2} \cos ^{2} \theta>1$, only (and always) capillary waves are generated; if $y_{1, \min }<M^{2} \cos ^{2} \theta<1$, both capillary and gravity waves are generated; if $M^{2} \cos ^{2} \theta<y_{1}$, min (i.e. $M$ is relatively small), no spectral wave components are excited.

The latter case deserves special commentary. It is important to distinguish between the radiation of surface waves (which decay with distance as $1 / \sqrt{r}$ when $r \gg k^{-1}$ ) and the deformation of the free surface over the source (which decays faster than $1 / \sqrt{r}$ and thus is limited only to the near field). The fact that in the range of small $M$ no real valued solutions for $k$ exist means that no radiation is emitted, even though the local deformation always exists.

To explain the physical essence of the radiation effect, consider Fig. 1 (from Ginzburg (1996) where we corrected a small typo). Figure 1 illustrates a point source moving from point $A$ towards point $B$ with constant velocity $\boldsymbol{V}$. At point $A$ the source generates waves with broad spectral composition. Each component is described by the phase speed in the medium $c_{\boldsymbol{k}}$. By the time the source reaches point $B$ at distance $V t$, the cylindrically diverging wave from point $A$ propagates a distance $c_{k} t$. Next look at line $A-D$ in the direction of the wave vector $\boldsymbol{k}$ of a field spectral component. The phase difference $\Delta \psi(\omega)$ between cylindrical waves (asymptotically decaying with distance as $\left.\sim \exp \left(-i \omega_{k} t+i k r\right) / \sqrt{r}\right)$ generated at points $A$ and $B$, and observed at "infinity" along angle $\theta$ to the trajectory of the body, is given by the expression $\Delta \psi=k(D A-$ $C A)=k\left(V t \cos \theta-c_{k} t\right)$ because $\psi^{B}(\omega)=\psi^{C}(\omega)$. At large distances, we can neglect the difference between $r_{A \infty}$ and $r_{B \infty}$ when considering amplitudes, but for the phase relationships this distinction is essential. The waves do not cancel each other at infinity if $\Delta \psi=k\left(V \cos \theta-c_{\boldsymbol{k}}\right) t \ll \pi$. For any $t$, this condition is realized only for $\cos \theta=c_{k} / V$, i.e. when $V>c_{\boldsymbol{k}}>c_{\min }$. For surface waves $c_{\min } \sim 23 \mathrm{~cm} / \mathrm{s}$. 
The presence of the Dirac function permits us to calculate the integral (26) and to obtain an analytical expression for the angular power radiation ${ }^{4}$ :

$$
\begin{array}{r}
P(\theta)=\frac{V^{2}}{4 \pi}(\epsilon D)^{2} l_{\perp}^{2} \times \\
\times\left.\sum_{m=1}^{2} \frac{g_{k}}{\cosh ^{2} k D}\left|\partial_{k} \sqrt{k^{-1} g_{k} \tanh k D}\right|^{-1}\right|_{k=k^{(1,2)}(\theta)}
\end{array}
$$

In real conditions the parameter $\mu=k_{m} D \gg 1$ and factor $\cosh ^{2} k^{(2)} D \simeq \exp \left(-2 k_{m} D\right) \ll 1$ effectively suppress the capillary wave component contribution to the radiation power. This signifies that formally we can write $\sigma=0$ for all expressions and simplify estimations. In this case only one solution of Eq. (27) exists, $g_{k} \rightarrow g$, and the angular power of radiation is thus given by the simple expression

$P(\theta)=\frac{1}{2 \pi}(\epsilon D)^{2} l_{\perp}^{2}(g D)^{3 / 2} \Phi(\cos \theta)=P_{0} \Phi(\theta)$,

where

$\Phi(\cos \theta)=\left.M^{2} \frac{x^{3 / 2} \sqrt{\tanh x}}{\left|\frac{1}{2} \sinh 2 x-x\right|}\right|_{x=x(\cos \theta)}$

is the dimensionless power and may be called directivity function. Parameter $x$ satisfies the condition of resonance (27) when $g_{k}$ is replaced with $g$, and therefore

$$
\cos \theta=\frac{1}{M} \sqrt{\frac{\tanh x}{x}\left(1+\frac{x^{2}}{\mu^{2}}\right)} \simeq \frac{1}{M} \sqrt{\frac{\tanh x}{x}} \leq 1
$$

for $x \ll \mu$. For $x=k D \ll 1$, a solution of Eq. (32), exists only when $M \geq \sqrt{\tanh x / x}$ : for small $x \ll 1, M \geq 1$, and for large $x \gg 1, M \geq 1 / \sqrt{x}$. Note that these estimates are valid in case of $k \ll k_{m}$.

Numerical calculation of the radiation direction for values $M \equiv V / \sqrt{g D}=1.1,2,3$, and 4 are shown in Fig. 2.

Let us analyze expressions (31) and (32). The function $\Phi \simeq 3 M^{2} x^{-1}$ for small values $x \ll 1$. For large values $x \gg 1$, the function is exponentially small, $\Phi \sim 4 M^{2} x^{3 / 2} e^{-2 x}$, i.e. it can be neglected because the principal contribution to the radiated energy comes from the domain near $x \ll 1$. A quick approximation is thus $\Phi \simeq 3 M^{2} x^{-1} \Theta(1-x)$. On the other hand, for small $x$, from Eq. (32) we have $M^{2} \cos ^{2} \theta \simeq 1-\frac{1}{2} x^{2}$, i.e. $M^{2} \cos ^{2} \theta \sim 1$. We obtain hence that $x \simeq \sqrt{2\left(1-M^{2} \cos ^{2} \theta\right)} \simeq \sqrt{1-M^{4} \cos ^{4} \theta}$. These simple estimates let us obtain a quick approximation

$$
\begin{array}{r}
P(\theta) \simeq \frac{3}{2 \pi}(\epsilon D)^{2} l_{\perp}^{2}(g D)^{3 / 2} \times \\
\frac{M^{2}}{\sqrt{1-M^{4} \cos ^{4} \theta}} \Theta\left(1-\sqrt{1-M^{4} \cos ^{4} \theta}\right) .
\end{array}
$$

\footnotetext{
${ }^{4}$ It is useful to check the dimensions of the left and right parts of the expression. We have for the left part $[P(\theta)] \equiv[\dot{E}]=L^{5} T^{-3}$ and for the right part $\left[V^{2}\right]\left[D^{2}\right]\left[l_{\perp}^{2}\right]\left[g_{k}\right]\left[\left|\partial_{k} c_{p h}\right|^{-1}\right]=L^{2} T^{-2} \times L^{4} \times$ $L T^{-2} \times T L^{-2}=L^{5} T^{-3}$
}

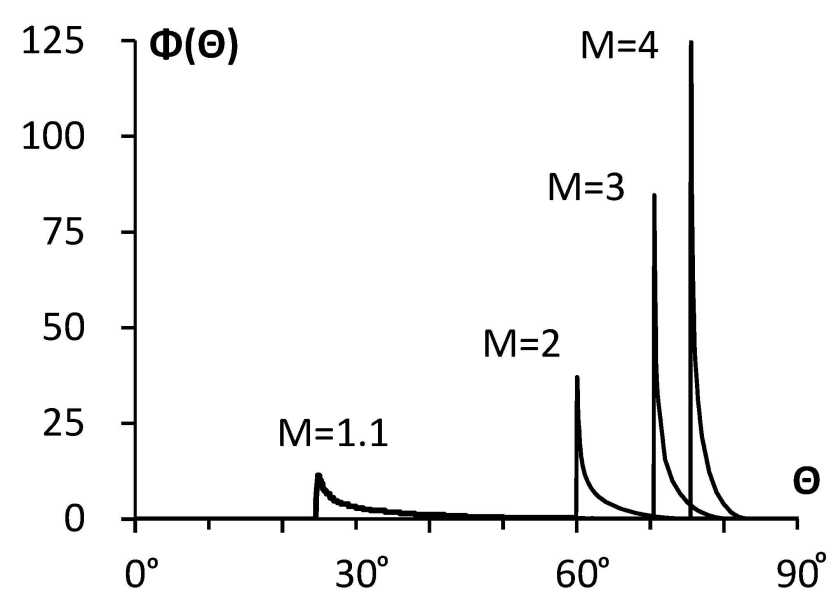

Fig. 2. Normalized power (directivity) of a tsunami, $\Phi(\theta)=P(\theta) / P_{0}$, as a function of the angle $\theta$ (measured from the direction of the rupture front propagation) for $M \equiv V / \sqrt{g D}=1.1,2,3$, and 4 .

In this expression - which is correct for the shallow-water approximation only $-D$ is the averaged ocean depth, $\epsilon D$ is the characteristic magnitude of the ocean floor perturbation, $g$ is the gravitational acceleration, and therefore, $\sqrt{g D}=c$ is the speed of the surface waves (the tsunami), $M=V / c$ is a parameter similar to the Mach number in acoustics: the ratio of source velocity and tsunami speed. If $V \gg c$, a "shock" surface wave can form in the direction of $\theta_{t s}=\arccos M^{-1}$.

\section{Radiation from a rupture front propagating with smoothly varying velocity}

Expression (13) for the spectral density of energy radiation,

$\left.E_{\boldsymbol{k}}\right|_{t \rightarrow \infty}=\omega_{\boldsymbol{k}}\left|\int_{-\infty}^{+\infty} d t^{\prime} e^{-i \omega_{\boldsymbol{k}} t^{\prime}} \frac{\delta H_{\text {int }}}{\delta a_{\boldsymbol{k}}}\right|^{2}$,

for arbitrary rupture front velocities is written as

$=\frac{g_{k}}{2(2 \pi)^{2}} \frac{\left|f_{k}\right|^{2}}{\cosh ^{2} k D}\left|\int_{-\infty}^{+\infty} d t^{\prime} e^{-i\left(\omega_{k} t^{\prime}-k \cdot x_{0}\left(t^{\prime}\right)\right.}\right|^{2}$.

Here, $f_{\boldsymbol{k}}$ is the form-factor of the "frozen" vertical bottom velocity in the frame of reference which travels with velocity $\boldsymbol{v}(t)=d \boldsymbol{x}_{0}(t) / d t$, which may be varying with time. The form-factor (17) is an experimentally measured quantity. Eq. (35) can be written as $E_{\boldsymbol{k}} \sim\left|L_{\boldsymbol{k}}\right|^{2}$ where

$L_{k}=\int_{-\infty}^{\infty} d t e^{i \omega_{k} t-i \boldsymbol{k} \cdot x_{0}(t)}$

is the amplitude of the process (or the amplitude of the radiative field of surface waves). $L_{k}$ is determined by the regime of the rupture front propagation $\mathbf{x}_{0}(t)$. The direction of vector $\boldsymbol{k}$ represents the direction of $\boldsymbol{k}$-surface wave 
mode radiation. Eq. (36) can be considered as the Fourier component of the function $\boldsymbol{v}(t) \exp \left[i \boldsymbol{k} \cdot \boldsymbol{x}_{0}(t)\right]$. This leads to some general conclusions. For example, if $\boldsymbol{x}_{0}(t)$ is an analytical function (all of its derivatives are continuous), then function $\boldsymbol{v}(t) \exp \left[i \boldsymbol{k} \cdot \boldsymbol{x}_{0}(t)\right]$ is analytical too. Therefore, based on the Titchmarsh's theorem (Titchmarsh, 1937) about Fourier transform properties, $L_{k}$ tends to zero faster than any integer power of $\omega$ with an increase of frequency $\omega$.

Let us produce the exact analytical result. Consider a rupture front moving with the velocity changing according to the following law

$\boldsymbol{v}(t)=\frac{\boldsymbol{v}_{2}+\boldsymbol{v}_{1}}{2}+\frac{\boldsymbol{v}_{2}-\boldsymbol{v}_{1}}{2} \tanh \frac{t}{\tau}$.

In Eq. (37), when time $t \rightarrow-\infty$, velocity $\boldsymbol{v}(t) \rightarrow \boldsymbol{v}_{1}$. When time $t \rightarrow+\infty$, velocity $\boldsymbol{v}(t) \rightarrow \boldsymbol{v}_{2}$. Transition from $\boldsymbol{v}_{1}$ to $\boldsymbol{v}_{2}$ occurs smoothly during the time interval of order $\tau$. Corresponding to Eq. (37) position of the front, $\boldsymbol{x}_{0}(t)$, is

$\boldsymbol{x}_{0}(t)=\frac{\boldsymbol{v}_{2}+\boldsymbol{v}_{1}}{2} t+\frac{\boldsymbol{v}_{2}-\boldsymbol{v}_{1}}{2} \tau \ln \cosh \frac{t}{\tau}$.

Substituting Eqs. (37) and (38) into Eq. (36), we find that

$L_{\boldsymbol{k}}=\int_{-\infty}^{\infty} d t e^{i\left(\omega_{\boldsymbol{k}}-\boldsymbol{k} \cdot \overline{\boldsymbol{v}}\right) t} \cosh ^{-i \frac{1}{2}(\boldsymbol{k} \cdot \Delta v) \tau} \frac{t}{\tau} \equiv \tau F(a, b)$.

Here, $a=\left(\omega_{\boldsymbol{k}}-\boldsymbol{k} \cdot \overline{\boldsymbol{v}}\right) \tau$ is the pulsation of the surface wave corrected for a Doppler factor, the parameter $b=(\boldsymbol{k} \cdot \Delta \boldsymbol{v}) \tau / 2$ describes the rate of front velocity change, $\Delta \boldsymbol{v}=\boldsymbol{v}_{2}-$ $\boldsymbol{v}_{1}, \overline{\boldsymbol{v}}=\left(\boldsymbol{v}_{2}+\boldsymbol{v}_{1}\right) / 2$. If $\Delta \boldsymbol{v} \rightarrow 0$, the integral degenerates into a delta-function

$F(a, 0) \rightarrow 2 \pi \delta(a)$.

The integral $F(a, b)$ can be calculated analytically:

$$
\begin{gathered}
F(a, b)=\int_{-\infty}^{\infty} d s \frac{e^{i a s}}{\cosh ^{i b} s} \equiv 2^{i b} \int_{0}^{\infty} d t \frac{t^{i(a+b)-1}}{\left(1+t^{2}\right)^{i b}}= \\
=2^{i b-1} \frac{\Gamma\left(\frac{1}{2}(i b-i a)\right) \Gamma\left(\frac{1}{2}(i b+i a)\right)}{\Gamma(i b)},
\end{gathered}
$$

where $\Gamma(s)$ is Euler's Gamma-function ${ }^{5}$. Using the identity $|\Gamma(i y)|^{2}=\pi / y \sinh \pi y$ with $\Im y=0$ (Abramovitz and Stegun, 1964; Gradshtein and Ryzhik, 1971), we can find the exact analytical expression for the radiated spectral power of surface waves for the parameters $a, b \neq 0$ and analyze limiting cases that present physical interest:

$|F(a, b)|^{2}=\pi \frac{b \sinh \pi b}{\left(a^{2}-b^{2}\right) \sinh \frac{\pi}{2}(a-b) \sinh \frac{\pi}{2}(a+b)}$.

Figure 3 shows the distribution $|F(a, b)|^{2} \propto E_{\boldsymbol{k}}$ for $-4.5<a<4.5$ and $-5<b<5$. If $\mathbf{v}_{1}=\mathbf{v}_{2}(b=0)$, radiation exists only for condition $a=0$. When $b \neq 0$ (i.e. the rupture front

\footnotetext{
${ }^{5}$ Integral (41) should be understood as a limit of the expression $\lim _{\epsilon \rightarrow 0} 2^{i b+\epsilon-1} \Gamma\left(\frac{1}{2}(i b+\epsilon-i a)\right) \Gamma\left(\frac{1}{2}(i a+i b+\epsilon)\right) / \Gamma(i b+\epsilon)$, i.e. when small dissipation is introduced into the wave system.
}

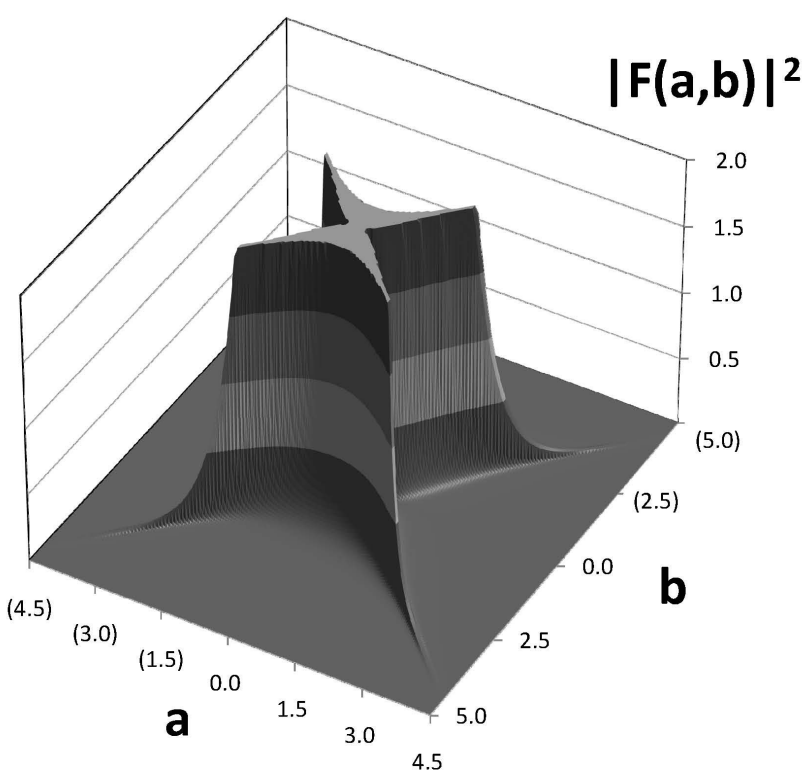

Fig. 3. Distribution of the dimensionless spectral energy of surface wave radiation $|F(a, b)|^{2}$. The parameter $a=\left(\omega_{\boldsymbol{k}}-\boldsymbol{k} \cdot \overline{\boldsymbol{v}}\right) \tau$ is the pulsation of the surface wave corrected for Doppler factor, parameter $b=(\boldsymbol{k} \cdot \Delta \boldsymbol{v}) \tau / 2$ describes the rate of front velocity change. To reveal the cross-section of the distribution, the magnitude is sliced off at $|F(a, b)|^{2}=2.0$.

changes its velocity direction or magnitude), the main part of waves is emitted in directions defined by $a \simeq \pm b$. The spectrum falls off exponentially at the periphery of the distribution. This characteristic is common for relatively smooth trajectories.

\section{Conclusions}

In this paper we applied the Hamiltonian method, as an alternative to the traditional consideration, to the problem of tsunami generation by a moving rupture front. We focused on the methodology rather than the specific phenomenon. We aimed to develop the methodology that is applicable to a broad range of general cases.

Obviously, an earthquake generates all types of waves longitudinal and transverse waves in the crust, volume waves in the ocean, surface waves both in the ocean and the atmosphere, internal waves, etc. The traditional tsunami consideration - which focuses on the large-scale surface waves in the approximation of an incompressible fluid - is just an individual case of the general problem of wave generation in the full system which includes elastic Earth layer, liquid ocean, gaseous atmosphere, and even ionosphere (see, for example, Pavlov and Sukhorukov, 1987). The Hamiltonian methodology is capable of dealing with the complete general problem because it uses the method of "propagator" - an operator 
which translates the original "cause" into the resulting "effect". By definition, the propagator (the Green function) contains all the necessary information about the medium via a dispersion relation. Since the method describes the entire system at once, one of the obvious advantages is that the principle of causality does not get violated. The other advantage is that the approach automatically solves for all types of waves generated in the system by an earthquake. (Mathematically, this is accomplished by solving for the poles of the propagator - the Green function - which determines all dispersion branches. Then the full Hamiltonian includes the sum over all the branches. Because the densities of the Earth's crust, the ocean and the atmosphere differ greatly, the motion of the solid component appears practically independent.) But obviously the energy input into the different wave frequency domains varies greatly. For large spatial scales and low phase velocities the principal channels of radiation are the surface waves (tsunami) and internal waves. This does not mean, however, that other types of waves do not originate. They are also generated (Nosov, 2000; Gisler, 2008) but their intensity is small relative to the intensity of the tsunami, and their spectral domain is localized at higher frequencies. ${ }^{6}$

We illustrated how the Hamiltonian method is applied to derive analytical expressions describing two measurable characteristics - directivity $(\Phi(\theta))$ and power $\left(P_{0}\right)$ - of tsunami radiation caused by traveling perturbations of the ocean floor. To derive workable expressions, we considered two simple cases: a source moving with uniform velocity and a source moving with smoothly varying velocity. Only the form-factor of the bottom deformation, $f_{k}$, and the velocity of the floor rupture propagation, $\boldsymbol{v}(t)$, are needed to be input into the expressions to determine the power and the directivity of the tsunami. In the idealized case of a uniformly moving rupture, the principal energy contribution to radiation comes from the large-scale components of the surface wave field whose scale is defined by the horizontal scale of bottom deformation. In the framework of this approximation, intensive radiation of long waves takes place if the speed of the ocean floor deformations is sufficiently great,

\footnotetext{
${ }^{6}$ While small in absolute terms, the hydro-acoustical fields resulting from ocean floor earthquakes can still be sufficiently intense. There is evidence that some animals are able to hear fast acoustic waves. Thus, for example, it has been observed that jellyfish move away from the shore and into the open ocean before tsunami arrival (Shuleikin, 1968). In contrast, deep-water fish apparently rise up to surface. Tsunami catalogs have repeatedly mentioned that tsunami waves threw out deep-water fish onto the coast (Soloviev and Go, 1975). Numerous animals - elephants, buffalo, flamingos, rats, and others - have also been observed becoming agitated and running away from the shore onto higher ground many minutes, or even hours, prior to tsunami arrival. http://news.nationalgeographic. com/news/2005/01/0104_050104_tsunami_animals.html and http: //www.pbs.org/wnet/nature/episodes/can-animals-predict-disaster/ video-full-episode/268/
}

i.e. approximately $M>1(V>\sqrt{g D})$. If the Froude number is large, $V \geq \sqrt{g D}$, as a large-scale surface wave, a tsunami is generated essentially close to the direction of the propagating rupture, in the angle range $0 \leq \theta \leq \theta_{\max } \equiv \arccos M^{-1}$ with maximum near $\theta_{\max }$. When $V \gg \sqrt{g D}$, radiation takes place essentially quasi-perpendicularly to the propagation of the rupture front. This last case is probably the most typical in geophysical conditions $\left(D \sim 4 \mathrm{~km}, g \sim 10 \mathrm{~m} / \mathrm{s}^{2}\right)$, where the speed of propagation of the radiated tsunami $\sqrt{g H} \sim 200 \mathrm{~m} / \mathrm{s}$ is significantly smaller than the rupture speed, which is comparable to crustal seismic shear-wave speeds $(V \sim 2.5 \mathrm{~km} / \mathrm{s})$. Obviously, the statement about radiation directivity is correct only when we neglect dispersive small-scale effects and operate in the framework of linear shallow-water theory. Smallscale (low-energy capillary or gravity-capillary) waves are generated for any values of $M$. It is these components that are responsible for the fine structure of the radiated surface "shock" wave. The apparent non-convergence of the shallow-water approximation in the vicinity of the resonance $(M \rightarrow 1)$ is suppressed by several factors, not considered in this note: (small) existing dissipation in the real system (bottom friction, diffusion on bottom non-homogeneities), dispersive effects which violate the phasing conditions between superposing emitted waves, finite duration of the process that "smoothes" the delta-function, and others. Even though the rupture propagates faster than sound waves in water, the "shock" surface wave does not form because such a wave is a superposition of all emitted waves (including capillary and capillary-gravity waves) which originate from the entire trajectory of the source (Pavlov and Sukhorukov, 1985).

In conclusion, the analytical results produced in this paper can be used by experimental or numerical researchers. Thus, Eq. (33) can be verified directly using experimental data. One can account for the finiteness of the process duration by replacing the delta-function in Eq. (33): $\pi \delta(\Delta \omega) \rightarrow$ $\tau /\left[1+(\tau \Delta \omega)^{2}\right]$ with $\epsilon \rightarrow \epsilon \exp (-t / \tau) \theta(t)$.

The exact analytical solution found for the special case of smoothly varying trajectories can be easily modified to the bounded time-spacial domain and serve as a useful benchmark for testing numerical models. This is accomplished by setting finite limits in Eq. (20). Then the delta-function transforms into a "spread" delta-function. But if the duration of the process is much longer than the characteristic period of the generated waves, the difference between the two is not significant and the overall tendency - the directivity of radiation - will be captured correctly.

\section{Appendix A}

\section{Validation of incompressible ocean assumption}

When is the incompressible ocean assumption valid in consideration of wave processes? 
Let us formulate a qualitative criterion for when fluid can be considered incompressible (we pattern our derivation partially after Landau (1965); Landau and Lifshitz (1987) and add estimates for the non-stationary process). The continuity condition $\partial_{t} \rho+\operatorname{div} \rho \boldsymbol{v}=0$ can be written as $\operatorname{div} \boldsymbol{v}=-\left(\rho c^{2}\right)^{-1} d p / d t$ if the evolution process is admitted adiabatic (entropy $s$ of mass unit is constant). Here, the partial derivative of pressure, $p$, with respect to fluid density, $\rho$, defines the sound speed in fluid, $c$ : $c^{2}=\left.\partial_{\rho} p\right|_{s}$. The full derivative with respect to time is given by $d p / d t=\partial_{t} p+v \cdot \nabla p$. The magnitude of the flow velocity is $V$, and characteristic variations of field variables in space and at time, realized at the characteristic scales $L$ and $T$, are defined as $\Delta V, \Delta p$, etc. (It is important to distinguish $V$ and $\Delta V$.) Now we can evaluate the second term in the continuity equation. We obtain $\left|-\left(\rho c^{2}\right)^{-1} d p / d t\right| \sim$ $\left(\rho_{0} c^{2}\right)^{-1} \max (\Delta p / T, V \Delta p / L) \sim \Delta p / \rho_{0} c^{2} \max (1 / T, V / L)$. Here, the expression $\max (x, y)$ denotes that a maximal value is chosen from the pair $x$ and $y$. Another equation - the momentum conservation equation - permits to evaluate $\Delta p$. In fact, the equation $-\rho^{-1} \nabla p=\partial_{t} \boldsymbol{v}+(\boldsymbol{v} \cdot \nabla) \boldsymbol{v}$ gives the estimate $\Delta p / \rho_{0} L \sim \max (\Delta V / T, V \Delta V / L)$. From here, we find that $\Delta p \sim \rho_{0} L \Delta V \max (1 / T, /, V / L)$. From these two expressions, it follows that $\left|-\left(\rho c^{2}\right)^{-1} d p / d t\right| \sim L c^{-2} \Delta V \max \left(1 / T^{2}, V^{2} / L^{2}\right) . \quad$ On the other hand, $|\operatorname{div} v| \sim \Delta V / L$. Comparing the left and right terms in the continuity equation, $\operatorname{div} v=-\left(\rho c^{2}\right)^{-1} d p / d t$, we can conclude that the second term can be neglected when $\Delta V / L \gg L \Delta V / c^{2} \max \left(1 / T^{2}, V^{2} / L^{2}\right)$, i.e. $L^{2} / c^{2}$ $\max \left(1 / T^{2}, V^{2} / L^{2}\right)=\max \left(L^{2} / c^{2} T^{2}, V^{2} / c^{2}\right) \ll 1$.

This expression produces the desired answer: a fluid can be considered incompressible when simultaneously the flow velocity (i.e., the amplitude of the vibration velocity of liquid particles, not the speed of the wave) is small with respect to the sound speed, $V^{2} \ll c^{2}$, and when characteristic space and time scales satisfy the condition $L^{2} \ll c^{2} T^{2}$. In this case, instead of the exact continuity condition, one can use the approximation $\operatorname{div} \boldsymbol{v}=0$.

For tsunami and other seismic-type periodical wave processes, velocities of fluid particles are typically small, $V \ll c$. The first condition is then satisfied. The second condition, which can be rewritten in form $L^{2} / T^{2} \ll c^{2}$, physically signifies that if in the process of wave generation, wave modes of spatial scale $L$ are excited, they are characterized by the time scale $T(L)$ following from the dispersion relationship for these modes. Thus, the relationship $L / T$ defines the phase velocity for this type of waves, $c_{p h}$, and the condition $L^{2} / T^{2} \ll c^{2}$ makes transparent physical sense: perturbations of scale $L$ propagating with smaller speed $c_{p h}(L)$ than the sound speed, $c_{p h}(L) \ll c$, can be considered as perturbations propagating in incompressible fluid. This is always valid in case of a tsunami for which the speed of propagation $c_{p h}(L) \simeq \sqrt{g D} \ll c$ regardless of the velocity of the traveling source.

\section{Appendix B Nonlinear effects}

In this paper we considered, in the linear approximation, radiative effects caused by a propagating rupture front underneath the ocean. However, when the amplitude of the radiated waves is sufficiently large, or when the waves have traveled sufficiently large distances, accumulated nonlinear effects have to be taken into account. In the framework of the formulated method, these nonlinear effects are captured in the evolution equations by the (non-zero) Hamiltonian $H_{\text {int }}$ which describes wave interactions and field excitation. The presence of $H_{\text {int }}$ leads to the appearance of additional terms in the wave equation.

To illustrate the point, let us consider the manifestation of nonlinear effects in the framework of a slowly varying wave train for the surface displacement which is described by Eq. (7)

$\eta(\boldsymbol{x}, t)=\int \frac{d \boldsymbol{k}}{2 \pi} \sqrt{\frac{\omega_{\boldsymbol{k}}}{2 g_{k}}}\left(b_{\boldsymbol{k}} e^{-i \omega_{\boldsymbol{k}} t}+b_{-\boldsymbol{k}}^{*} e^{+i \omega_{-\boldsymbol{k}} t}\right) e^{i \boldsymbol{k} \cdot \boldsymbol{x}}$.

Here, $\omega_{\boldsymbol{k}}=\sqrt{k g_{k} \tanh k D}$ is the dispersion law for free waves, $\mathbf{k}$ is the wave vector, $D$ is the thickness of the water layer, $g_{k}=g+\sigma k^{2}, g$ is the gravitational acceleration, $\sigma$ is the surface tension coefficient. The normal variables $b_{\boldsymbol{k}}, b_{\boldsymbol{k}}^{*}$ satisfy the nonlinear equations

$\partial_{t} b_{\boldsymbol{k}}=-i \frac{\delta H_{\mathrm{int}}}{\delta b_{\boldsymbol{k}}^{*}}, \quad \partial_{t} b_{\boldsymbol{k}}^{*}=+i \frac{\delta H_{\mathrm{int}}}{\delta b_{\boldsymbol{k}}}$,

which result from Eq. (10) and where $H_{\text {int }}$ includes both terms describing wave interactions and terms describing the field excitation. Suppose that the conditions of field formation are such that the significant contribution comes only from a small range of wave-numbers and frequencies near $\boldsymbol{k}_{0}$ and $\omega_{0}$ corresponding to the carrier wave. Then the surface displacement, Eq. (B1), can be written as

$$
\begin{gathered}
\eta(\boldsymbol{x}, t) \simeq e^{-i\left[\omega_{0} t-\boldsymbol{k}_{0} \cdot \mathbf{x}\right]} \times \\
\sqrt{\frac{\omega_{0}}{2 g}} \int \frac{d \boldsymbol{\kappa}}{2 \pi}\left(b_{\boldsymbol{k}} e^{-i\left[\left(\omega_{\boldsymbol{k}}-\omega_{0}\right) t-\boldsymbol{\kappa} \cdot \boldsymbol{x}\right]}\right. \\
+ \text { c. c. }(\boldsymbol{k} \rightarrow-\boldsymbol{k}),
\end{gathered}
$$

specifying a wave train with a local (envelope) amplitude of $A=\sqrt{\frac{\omega_{0}}{2 g}} \int \frac{d \boldsymbol{\kappa}}{2 \pi} b_{\boldsymbol{k}} e^{-i \psi}$.

Here, $\boldsymbol{\kappa}=\boldsymbol{k}-\boldsymbol{k}_{0}, \quad \psi=\left(\omega_{\boldsymbol{k}}-\omega_{0}\right) t-\boldsymbol{\kappa} \cdot \boldsymbol{x}$. Because the wave interactions are weak but non-negligible $\left(H_{\text {int }} \neq 0\right)$, the normal amplitude of an individual wave component $b_{\boldsymbol{k}}$ is a slowly varying function of time. Therefore, the rate of change of the local amplitude is

$\partial_{t} A=\sqrt{\frac{\omega_{0}}{2 g}} \int \frac{d \boldsymbol{\kappa}}{2 \pi}\left[\dot{b}_{\boldsymbol{k}} e^{-i \psi}-i\left(\omega_{\boldsymbol{k}}-\omega_{0}\right) b_{\boldsymbol{k}} e^{-i \psi}\right]$.

Since the wave spectrum is narrow, frequency $\omega_{\boldsymbol{k}}$ can be expanded about $\boldsymbol{k}_{0}$, giving

$\omega_{\boldsymbol{k}}-\omega_{0}=\kappa_{i}\left(\partial_{k_{i}} \omega\right)_{0}+(1 / 2) \kappa_{i} \kappa_{j}\left(\partial_{k_{i}} \partial_{k_{j}} \omega\right)_{0}+\ldots$. 
Having in mind that

$\partial_{i} A=i \sqrt{\frac{\omega_{0}}{2 g}} \int \frac{d \kappa}{2 \pi} \kappa_{i} b_{\boldsymbol{k}} e^{-i \psi}$,

and $\quad \partial^{2} \omega / \partial k_{i} \partial k_{j}=\left(v_{g} / k\right)\left(\delta_{i j}-k_{i} k_{j} / k^{2}\right)+\left(v_{g}^{\prime} / k^{2}\right) k_{i} k_{j}$, Eq. (B5) for the envelope simplifies to

$$
\begin{array}{r}
\left(\partial_{t}+v_{g} \partial_{x}\right) A+i \frac{v_{g}^{\prime}}{2} \partial_{x x} A+i \frac{v_{g}}{2 k_{0}} \Delta_{\perp} A= \\
=-i \sqrt{\frac{\omega_{0}}{2 g}} \int \frac{d \boldsymbol{\kappa}}{2 \pi} \frac{\delta H_{\text {int }}}{\delta b_{\boldsymbol{k}}^{*}} e^{-i \psi} .
\end{array}
$$

Here, $\boldsymbol{v}_{g}=\left(\partial_{\boldsymbol{k}} \omega\right)_{0}$ is the group speed, $\boldsymbol{v}_{g}^{\prime}$ is the derivative of the group speed module with respect to $k$, the first two terms on the left hand side of the equation represent the envelope propagation along the $x$-axis with the group speed $v_{g}$, the next terms on the left represent the effects of linear longitudinal and transversal dispersion about the carrier wave-number and frequency with the finite but small spectral band-width. All terms in the left part of the equation describe only linear propagation effects. All information about interactions and excitation is contained in the final term of Eq. (B8) for which $\dot{b}_{\boldsymbol{k}}=\left\{b_{\boldsymbol{k}}, H_{\text {int }}\right\} \equiv-i \delta H_{\text {int }} / \delta b_{\boldsymbol{k}}^{*} \neq 0$ where the bracket defines the functional Poisson bracket. Calculations of the functional derivative of type $\delta H_{\text {int }} / \delta b_{k}^{*}$ (expressed in terms of $A, A^{*}$ ) for a specific model can be accomplished using standard procedures.

The advantages of the Hamiltonian approach for wave and vortex dynamics are now well-established (see for example Goncharov and Pavlov (1997, 1998, 2008) where a numerous bibliography is given). In particular, for wave processes in Hamiltonian description, specific features of the medium are fully contained in the dispersion relation; all versions of perturbation theory are considerably simplified and standardized; the results of calculations obtained for a particular medium are easily interpreted and assigned physical meaning. One of the consequences of the Hamiltonian approach is that the integro-differential evolution equations are expressed in a standard form for the so-called normal variables $\left(a, a^{*}\right)$ which are related to the "natural" physical variables via Fourier's transformations. The general structure of these nonlinear equations is the same for waves of different physical nature in nonlinear dispersive media. From these evolution equations for normal variables, one usually derives somewhat simpler integro-differential equations (called "simplified equations") for auxiliary variables. The simplified equations usually serve as a starting point for the study of wave instabilities, long-time wave evolution, derivation of transfer equation for spectrum of random wave field, and for many other applications.

Edited by: S. Tinti

Reviewed by: two anonymous referees

\section{References}

Abramovitz, M. and Stegun, I. A.: Handbook of Mathematical Functions, US Govt. Printing Office, Washington DC, USA, 1964.

Ammon, C. J., Ji, C., Thio, H. K., Robinson, D., Ni, S. D., Hjorleifsdottir, V., Kanamori, H., Lay, T., Das, S., Helmberger, D., Ichinose, G., Polet, J., and Wald, D.: Rupture process of the 2004 Sumatra-Andaman earthquake, Science, 308, 1133-1139, 2005.

Bryant, E.: Tsunami: The Underrated Hazard, Cambridge University Press, Cambridge, UK, 320 pp., 2001.

Dahlen, F. A. and Tromp, J.: Theoretical Global Seismology, Princeton University Press, Princeton, NJ, USA, 1998.

Ginzburg, V. L.: Radiation by uniformly moving sources (Vavilov - Cherenkov effect, transition radiation, and other phenomena), Physics-Uspekhi, 39(10), 973-982, 1996.

Gisler, G. R.: Tsunami simulations, Annu. Rev. Fluid Mech., 40, 71-90, 2008.

Goncharov, V. P. and Pavlov, V. I.: Effets hydrodynamiques et leurs description hamiltonienne (Problemy gidrodinamiki v gamiltonovskom opisanii), Moscow Lomonossov State University, 1993.

Goncharov, V. P. and Pavlov, V. I.: Some Remarks on the Physical Foundation of the Hamiltonian Description of Fluid Motions, Eur. J. Mech., B/Fluids, 16(4), 509-555, 1997.

Goncharov, V. and Pavlov, V.: On the Hamiltonian approach: Applications to geophysical flows, Nonlin. Processes Geophys., 5, 219-240, 1998, http://www.nonlin-processes-geophys.net/5/219/1998/.

Goncharov, V. and Pavlov, V.: Large-scale vortex structures in shear flows, Eur. J. Mech., B/Fluids, 19, 831-854, 2000.

Goncharov, V. and Pavlov, V.: Multipetal Vortex Structures in Two - Dimensional Models of Geophysical Fluid Dynamics and Plasma, J Exp. Theor. Phys., 92, 594-607, 2001.

Goncharov, V., and Pavlov, V.: Hamiltonian vortex and wave hydrodynamics, GEOS, Moscow, 2008 [in Russian].

Gradshtein, S. and Ryzhik, L. M.: Tables of Integrals, Sums, Series and Products, Nauka, Moscow, 1971.

Harbitz, C. B., Løvholt, F., Pedersen, G., and Masson, D. G.: Mechanisms of tsunami generation by submarine landslides: a short review, Norw. J. Geol., 86, 255-264, 2006.

Hatori, T.: in Tsunamis in the Pacific Ocean, Honolulu, 60(21), 1970.

Ivanov, V. V.: Evolution of earthquake processes, Sov. Phys. Uspekhi, 34(3), 230-249, 1991.

Janssen, P.: The interaction of ocean waves and wind, Cambridge University Press, Cambridge/New York, 2004.

Kanamory, H. and Brodsky, E. E.: Physics of earthquakes, Reports on Progress in Physics, 67, 1429-1496, 2004.

Keating, B. H., Waythomas, C. F., and Dawson, A. G.: Landslides and tsunamis, Pageoph Topical Volumes, Birkhausser Verlag, Berlin, Germany, 157, 1313, 2000.

Kervella, Y., Dutykh, D., and Dias, F.: Comparison between threedimensional linear and nonlinear tsunami generation models, Theoretical and computational fluid dynamics, 21, 245-269, 2007.

Landau, L. D.: Collected Papers of L. D. Landau, edited by: ter Haar, D., Pergamon Press, Oxford/New York, 836 pp., 1965.

Landau, L. D. and Lifshitz, E. M.: Fluid Mechanics, Pergamon, Oxford, 1982. 
Landau, L. D. and Lifshitz, E. M.: Fluid Mechanics, V. 6 of Course of Theoretical Physics, 2nd English edition, 1987, $\$ 10$.

Lavrenov, I. V.: Wind-waves in oceans : dynamics and numerical simulations, Springer, New York, USA, 2003.

Murty, T.: Seismic sea waves - Tsunamis, B. Fish. Res. Boand Canada, 198, Ottawa, ON, Canada, 1977.

Novikova, L. E. and Ostrovsky, L. A.: Excitation of tsunami wavs by a traveling displacement of the ocean bottom, Marine Geodesy, 2, 365-380, 1979.

Nosov, M. A.: On the directivity of dispersive tsunami waves excited by piston-type and traveling-wave sea-floor motion, Volcanology and Seismology, 19, 837-844, 1998.

Nosov, M. A.: Tsunami generation in a compressible ocean by vertical bottom motions, Izvestiya, Atmos. Ocean. Phys., 36, 661669, 1999.

Nosov, M. A.: Tsunami generation in a compressible ocean by vertical bottom motions, Izvestiya, Atmos. Ocean. Phys., 36(5), 718-726, 2000.

Pavlov, V. I. and Slabeycius, J.: Damping of the surface wave due to the radiation of waves into the atmosphere, Izvesiya academii nauk SSSR, Fizika atmosphery i oceana, 19(5), 554-557, 1983.

Pavlov, V. I. and Sukhorukov, A. I.: Emission of acoustic transition waves, Sov. Phys. Usp., 28, 784-804, 1985.

Pavlov, V. I. and Sukhorukov, A. I.: Migrating ionospheric disturbances caused by spreading tsunami waves in ocean, Pisma v zhurnal tekhnicheskoi fiziki, 12(6), 351-354, 1987.

Pelinovsky, E. N.: Tsunami Wave Hydrodynamics, Institute Applied Physics Press, Nizhny Novgorod, 1996 (in Russian).

Pelinovsky, E. N.: Nonlinear models of tsunami generation by moving sources, in Nonlinear Waves 2002, Applied Physics Institute Press, Nizhny Novgorod, 199-210, 2003 (in Russian).

Pelinovsky, E., Talipova, T., Kurkin, A., and Kharif, C.: Nonlinear mechanism of tsunami wave generation by atmospheric disturbances, Nat. Hazards Earth Syst. Sci., 1, 243-250, 2001, http://www.nat-hazards-earth-syst-sci.net/1/243/2001/.

Shuleiikin, V. V.: Physics of the Sea (Fizika Morya), Nauka, Moscow, 1968 (in Russian).
Soloviev, S. L. and Go, Ch. N.: A catalogue of tsunamis on the eastern shore of the Pacific Ocean, Moscow, "Nauka" Publishing House, a) 308 pp., 1974; b) 202 pp., 1975, English translation: Soloviev, S. L. and Go, Ch. N.: A catalogue of tsunamis on the eastern shore of the Pacific ocean, Translation by Canada Institute for Scientific and Technical Information, National Research Council, Ottawa, Canada KIA OS2, 1984.

Sretensky, L. N.: Theory of wave motions of liquids, Nauka, Moscow, 1977.

Tanioka, Y. and Satake, K.: Tsunami generation by horizontal displacement of ocean bottom, Geophys. Res. Lett., 23, 861-864, 1996.

Tinti, S. and Bortolucci, E.: Analytical investigation of tsunamis generated by submarine slides, Annali di Geofisica, 43, 519-536, 2000 .

Tinti, S., Bortolucci, E., and Chiavettieri, C.: Tsunami excitation by submarine slides in shallow-water approximation, Pageoph., 158, 759-797, 2001.

Titchmarsh, R.: Introduction to the Theory of Fourier integrals, Clarendon Press, Oxford, 1937.

Titov, V. V., Rabinovich, A. B., Mofjeld, H. O., Thomson, R. E., and Gonzalez, F. I.: The global reach of the 26 December 2004 Sumatra tsunami, Science, 309, 2045-2048, 2005.

Ward, S. N. and Asphaug, E.: Asteroid impact tsunami of 2880 March 16, Geophys. J. Int., 153, F6-F10, 2003.

Wiegel, R. L.: Tsunami information sources 2006, Science of Tsunami Hazards, 24(2), 2006.

Zakharov, V. E. and Filonenko, N. N.: The energy spectrum for stochastic oscillation of a fluid's surface, Doklady Akad. Nauk, 170, 1292-1295, 1966.

Zakharov, V. E. and Kuznetsov, E. A.: Hamiltonian formalism for nonlinear waves, Physics - Uspekhi, 40(11), 1087-1116, 1997.

Zakharov, V. E.: Statistical theory of gravity and capillary waves on the surface of a finite-depth fluid, Eur. J. Mech., B/Fluids, 18(4), 527-344, 1999. 